\title{
Potência aeróbica máxima, freqüência cardíaca e capacidade vital em ambientes normo e hiperbárico
}

\author{
Carlos Alberto Moreira ${ }^{1}$ e Estélio Henrique M. Dantas²
}

\section{RESUMO}

Este estudo se propôs verificar variáveis fisiológicas. Revisando a literatura sobre o presente trabalho, levantaram-se vários tratados especificando as reações e adaptações tanto fisiológicas quanto comportamentais ocorridas no organismo durante o mergulho. Seguiu-se o modelo da pesquisa descritiva do tipo survey, da qual participaram mergulhadores da Marinha do Brasil, do sexo masculino, praticantes de atividades físicas num total de 9, com média da idade de $28 \pm 4,3$ anos, peso de $76,7 \pm 8,32 \mathrm{~kg}$ e estatura de $173,8 \pm 6,33 \mathrm{~cm}$.

Todos foram submetidos à avaliação de vários parâmetros fisiológicos determinados para o estudo ao nível do mar (ambiente normobárico) e aos 18 metros de profundidade (ambiente hiperbárico), pressurizados em câmara hiperbárica, sendo verificadas as variáveis propostas em exercício.

Os resultados são analisados no nível de significância de $\mathrm{p}$ $<0,05$, estabelecido como parâmetro neste estudo. A frequiência cardíaca $(\mathrm{p}=0,1468 ;>0,05)$ denota não existir diferença significativa entre os ambientes verificados. O consumo máximo de oxigênio ( $\mathrm{p}=0,00013 ;<0,05)$ e a capacidade vital $(\mathrm{p}=0,00126 ;<0,05)$ denotam existir diferenças significativas entre os ambientes. Isto comprova a importância de conhecer as reações fisiológicas avaliadas e revisadas na literatura como componentes essenciais da performance, com ênfase na fisiologia do exercício hiperbárica.

Palavras-chave: Fisiologia hiperbárica. Potência aeróbica máxima. Capacidade vital. Freqüência cardíaca. Medicina Esportiva.

1. Fisiologista e Gerontólogo; Mestre em Ciência da Motricidade Humana; Professor da Universidade Castelo Branco (UCB/RJ), Universidade Federal Fluminense (UFF/RJ) e Universidade Salgado de Oliveira (Universo/RJ).

2. Professor Titular do Programa de Mestrado em Ciência da Motricidade Humana da Universidade Castelo Branco - RJ.

Endereço para correspondência:

Carlos Alberto Moreira

Rua Curitiba, 178

24456-620 - São Gonçalo, RJ

Tel. (021) 603-2389 (residência)

(021) 9152-4299 (celular)

E-mail: poumo@rj.sol.com.br

\section{ABSTRACT \\ Maximum aerobic power, heart rate, and vital capacity in normo and hyperbaric environments}

This paper aims at studying physiological variables related to diving. The literature review on the subject found both physiological and behavioral reactions developed by the organism during diving. This research follows the survey model and investigated 9 divers belonging to the Brazilian Navy. They are all male; mean age $28 \pm 4.3$ years; weight $76.7 \pm 8.32 \mathrm{~kg}$; height $173.8 \pm 6.33 \mathrm{~cm}$. All subjects were evaluated using various physiological parameters, determined for sea level study (normobaric environment) and for 18 meters of depth (hyperbaric environment), pressurized in hyperbaric chamber, following variables proposed for exercises. The significance value established as a parameter is $p<0.05$. Heart rate $(p=0.1468 ;>0.05)$ showed there were no significant differences between the verified environments. Maximum oxygen consumption ( $p=0.00013 ;<0.05)$ and the vital capacity $(p=0,00126 ;<0.05)$ denoted significant differences between environments. The data confirmed the importance of knowing the physiological reactions evaluated and reviewed in the literature as essential components of performance with emphasis upon the physiology of hyperbaric exercise.

Key words: Hyperbaric physiology. Maximum aerobic power. Vital capacity. Heart rate. Sports Medicine.

\section{INTRODUÇÃO}

A gênese das atividades hiperbáricas associa-se fortemente às necessidades e vontades do homem em conduzir operações militares ou de salvamento, em conseguir alimentos e em expandir as fronteiras do conhecimento através da exploração e da pesquisa ${ }^{1,2}$.

Essas condições adversas oferecidas pelo meio ambiente levam os pesquisadores ao estudo das reações fisiológicas do organismo humano e à busca de equipamentos que supram as necessidades do homem durante sua incursão no mundo submarino $^{3}$, regido por leis diferenciadas e uma física toda especial $^{4,5}$. Nessa busca constante, o homem tenta adaptar-se a uma série de condições diferentes daquelas em que habitualmente vive. 
A aventura humana através dos mares desde a antiguidade encontra uma série de elementos adversos relativos às variações de pressão atmosférica, onde as diversas funções do organismo são variadamente influenciadas pelas condições alteradas do meio ambiente ${ }^{6}$.

Cabe enfatizar que as variações físicas e alterações fisiológicas citadas em vários estudos são diferenciadas pelo biótipo e adaptabilidade funcionais ocorridas pela interferência da pressão exercida durante o mergulho ${ }^{7}$. Os estudos mais recentes incluem as reações psicofisiológicas ocasionadas pelo meio ambiente ${ }^{8}$.

Nos dias atuais, a ênfase alterou-se em larga escala para um entendimento dos fatores que exercem efeito na performance do mergulhador. Com essa mudança, a função da medicina hiperbárica também mudou. Antes a preocupação era com os fatores fisiológicos, entretanto, afirma Bennet, citado por Bove \& Davis ${ }^{9}$. Porém agora, mais do que isso, um estudo dos elementos cruciais na boa performance humana no modo subaquático, o conhecimento dos especialistas da medicina hiperbárica, teve que se expandir, incluindo fatores como: água fria, descompressão, respiração de misturas gasosas e elementos emocionais (pânico, ansiedade) na performance do mergulhador. No entanto, a orientação fisiológica deverá incluir o campo psicofisiológico e as funções de tratamento e enquadramento em novas dimensões.

Numa perspectiva atual, busca-se uma união da "fisiologia do exercício - medicina do esporte" com a "medicina hiperbárica", na busca de novos conhecimentos aplicando-os na melhoria da performance dos mergulhadores.

\section{OBJETIVO}

O propósito deste estudo foi avaliar a influência do ambiente hiperbárico (aumento da pressão) sobre os parâmetros fisiológicos do organismo humano (freqüência cardíaca - FC, consumo máximo de oxigênio - $\mathrm{VO}_{2 \text { máx }}$ e capacidade vital CV).

\section{MATERIAL E MÉTODOS}

O presente estudo desenvolve-se utilizando o delineamento da "pesquisa descritiva" do tipo survey, que, segundo Flegner et al. ${ }^{10}$, é usada para comparar condições com critérios predeterminados ou para a avaliação da eficiência dos programas na busca de um perfil.

Os sujeitos selecionados para este estudo pertencem ao sexo masculino, saudáveis e aptos ao exercício da atividade de mergulho, sendo a idade mínima da amostra de 20 e a máxima de 40 anos. Todos os sujeitos são voluntários, integrantes do Grupo de Mergulhadores Militares da Base Almirante Castro e Silva - Marinha do Brasil, praticantes de atividades físicas (Treinamento Físico Militar - TFM), perfazendo um total de 9 , selecionados através dos critérios de inclusão e exclusão e assinam o termo de consentimento.
Tratando-se de uma pesquisa descritiva do tipo survey, deve obedecer uma sequiência de procedimentos para aplicação.

1) Avaliação do $V \mathbf{V}_{2 \text { máx }}: O$ protocolo de avaliação do $\mathrm{VO}_{2 \text { máx }}$ escolhido, o "Método de Balke"11,12, emprega cargas progressivas de 25 watts a cada intervalo de tempo de 2 minutos, caso o indivíduo não seja atleta, e 50 watts, caso seja atleta ou bem condicionado. $\mathrm{O}$ consumo de oxigênio pode ser estimado através da fórmula do American College of Sports Medicine ${ }^{13}$, verificando o peso corporal do avaliado antes da realização do teste, bem como a última carga completada pelo indivíduo em watts.

A aplicação do protocolo foi abordada de forma cruzada; parte da amostra (5) iniciou-se com o teste ao nível do mar e outra parte (4) na câmara. A intensidade do exercício é acompanhada a cada minuto através do índice de percepção subjetiva do esforço - Escala de Borg - modificada.

2) Avaliação da freqüência cardíaca: A frequiência cardíaca é monitorizada no ambiente de teste através de monitor por telemetria, modelo interface polar no 2390018 , que utiliza um sinal do peito para um relógio portátil de pulso que exibe a freqüência de acordo com a intensidade do exercício.

3) Avaliação da capacidade vital: A capacidade vital foi avaliada nos vários ambientes, onde o avaliado se encontra sentado (alterando a posição em função do espaço interno da câmara que não permite ao indivíduo permanecer de pé), frente ao espirômetro, devendo seguir as seguintes etapas: a) obstruir as narinas para que o ar não saia pelo nariz; b) realizar uma inspiração máxima; c) colocar o bocal do espirômetro bem ajustado na boca; e d) realizar uma expiração máxima, predita através do nomograma de Cournand, citado por Hollmann \& Hetinger ${ }^{14}$, sendo aferido por meio do espirômetro portátil ${ }^{15}$.

\section{INSTRUMENTAÇÃO}

Foram utilizados para este estudo os seguintes instrumentos: a) câmara hiperbárica tipo multiplace, com operador externo; b) interface polar $\mathrm{n}-2390018$; c) bicicleta atlanta; d) compasso de dobras cutâneas Harpenden; e) espirômetro portátil; f) balança portátil; e g) fita antropométrica e trena.

\section{APRESENTAÇÃO E DISCUSSÃO DOS RESULTADOS}

Para melhor compreensão dos dados colhidos, utilizam-se os procedimentos da estatística descritiva, no sentido de caracterizar o universo amostral. Todas as variáveis são apresentadas segundo os valores básicos da estatística.

A faixa etária do grupo pesquisado encontra-se de 20 a 40 anos e todos pertencem ao sexo masculino. A tabela 1 mostra as médias de idade, peso e altura dos participantes da pesquisa.

A tabela 2 mostra que existe diferença significativa $(\mathrm{p}=$ $0,00013 ;<0,05)$ no consumo máximo de oxigênio entre os 
valores médios do grupo analisado. Portanto, o ambiente hiperbárico altera o consumo máximo de oxigênio durante o teste de esforço.

Conforme apresentado na tabela 3, a freqüência cardíaca durante o teste de esforço ao nível do $\operatorname{mar}=171 \pm 10,9$, aos 18 metros $=164 \pm 11,2$.

Pode-se afirmar que não existe diferença significativa da freqüência cardíaca $(\mathrm{p}=0,1468 ;>0,05)$. Partindo destes dados, observa-se que a mesma não sofreu alterações significativas influenciadas pelo ambiente.

TABELA 1

Média, desvio-padrão, mínimo e máximo da amostra

\begin{tabular}{lclcc}
\hline \multicolumn{1}{c}{ Variável } & Média & DP & Mínimo & Máximo \\
& & & & \\
Idade (anos) & 28 & 4,3 & 23 & 36 \\
Peso $(\mathrm{kg})$ & 76,7 & 8,32 & 65,0 & 89,0 \\
Altura $(\mathrm{cm})$ & 173,8 & 6,33 & 164,5 & 182,0 \\
\hline
\end{tabular}

TABELA 2

Consumo máximo de oxigênio $\left(\mathrm{ml} \cdot \mathrm{kg}^{-1} \cdot \mathrm{min}^{-1}\right)$

\begin{tabular}{lllll}
\hline Variável & Média & DP & Mínimo & Máximo \\
latm & 46,7 & 4,34 & 41,2 & 55,5 \\
2,8 ata & 39 & 4,35 & 33 & 47 \\
\hline
\end{tabular}

TABELA 3

Freqüência cardíaca (bpm)

\begin{tabular}{lcccc}
\hline Variável & Média & DP & Mínimo & Máximo \\
latm & 171 & 10,9 & 156 & 192 \\
2,8 ata & 164 & 11,2 & 146 & 175 \\
\hline
\end{tabular}

TABELA 4

Capacidade vital (ml)

\begin{tabular}{lcccc}
\hline Variável & Média & DP & Mínimo & Máximo \\
Prevista & 4.229 & 148 & 4.040 & 4.440 \\
latm & 4.739 & 304,9 & 4.400 & 5.200 \\
2,8 ata & 4.644 & 332,1 & 4.300 & 5.200 \\
\hline
\end{tabular}

\begin{tabular}{lcccc}
\hline \multicolumn{5}{c}{$\begin{array}{c}\text { TABELA 5 } \\
\text { Apnéia (seg) }\end{array}$} \\
Variável & Média & DP & Mínimo & Máximo \\
latm & 120 & 33,8 & 57 & 161 \\
2,8 ata & 142 & 39,4 & 62 & 180 \\
\hline
\end{tabular}

Rev Bras Med Esporte - Vol. 5, № 5 - Set/ O ut, 1999
Constatou-se que existe diferença significativa entre os valores médios do grupo analisado, sendo o valor maior observado quando no ambiente de pressão igual a $1 \mathrm{~atm}$. Quando ao ambiente de 2,8 ata, observou-se uma diferença significante $(\mathrm{p}=0,00126 ;<0,05)$, em que a capacidade vital foi reduzida em função do ambiente em que estava exposto o indivíduo. Dessa forma, a mudança de pressão influenciou em 55,7\% na diferença entre as médias. De acordo com a literatura consultada, observou-se neste item o efeito direto da lei de Boyle ${ }^{16}$ sobre o organismo humano.

A tabela 5 mostra a média, desvio-padrão e amplitude da apnéia obtida ao nível do mar (1atm) e aos 18 metros $(2,8$ ata), em segundos. A média da apnéia ao nível do mar = 120; desvio-padrão $=33,8$; e a média aos 18 metros $=142$; desviopadrão $=39,4$. Seguindo o sistema analítico de comparações entre os valores médios da variável observada nas respectivas pressões de 1 atm e 2,8 ata, observou-se uma diferença significativa ( $\mathrm{p}<0,0229$; $<0,05$ ), sendo neste caso a média da apnéia observada em $1 \mathrm{~atm}<2,8$ ata e a mudança de pressão, respondendo por $27,6 \%$ da diferença entre as médias observadas nas respectivas pressões.

A extrapolação dessa variável, de acordo com as referências bibliográficas, ocorreu devido ao fato da dissolubilidade gasosa na pressão atmosférica aumentada, segundo a aplicabilidade direta da lei de Henry ${ }^{16}$.

\section{CONCLUSÕES}

Ao verificar, de acordo com os resultados obtidos através dos procedimentos estatísticos, as alterações fisiológicas provocadas pelo aumento da pressão atmosférica sobre o corpo humano, pode-se afirmar que ambas as variáveis - consumo máximo de oxigênio e capacidade vital - encontram-se influenciadas diretamente pelo devido ambiente. Quanto à freqüência cardíaca, constatou-se que o ambiente não influência diretamente, sendo a mesma influenciada pela intensidade e duração do exercício, independente do ambiente onde estiver sendo realizado.

Assim como o consumo máximo de oxigênio foi aumentado, a freqüência cardíaca também sofreu alteração. Ambos mantiveram uma relação linear com a carga de trabalho realizada, isto é, quanto maior a carga, maior o aumento no consumo de oxigênio e na freqüência cardíaca durante o teste para o mesmo indivíduo. Pela análise desses parâmetros e suas respostas no ambiente hiperbárico, houve mudanças com aumento do consumo de oxigênio. Fato corroborado pelas limitações impostas da ventilação e da massa muscular utilizada, influenciadas diretamente pela pressão atuante sobre o organismo. Comparando com a literatura ${ }^{17-19}$, constatou-se que a freqüência cardíaca durante o exercício submerso aumenta em relação ao trabalho executado em condições secas. Pode-se concluir que, diferente das equações para estimar o consumo máximo de oxigênio ao nível do mar, equações de confiança 
não têm sido desenvolvidas para o mergulho, onde interferências adaptativas fisiológicas ao estresse do meio, balanço térmico e pressão parcial de oxigênio exercem influências dire$\operatorname{tas}^{20}$. Mais detalhado, o uso de diferentes equipamentos de mergulho contribui ainda mais para complicar o uso de algoritmos singulares, especialmente para a freqüência cardíaca.

Mecanismos especiais de adaptação foram desenvolvidos pelos animais mergulhadores, que são parcialmente encontrados no homem em proporções bem menores. Esses mecanismos permitem a esses animais mergulharem por mais tempo do que um homem é capaz de mergulhar em apnéia. Num indivíduo treinado pode-se contar com um tempo de apnéia aproximado de 3 a 4 minutos, afirma a literatura. Este tempo, de acordo com os dados da pesquisa, poderá aumentar, uma vez que as moléculas gasosas são influenciadas pela pressão a que estão submetidas. Destaca-se que a concentração de oxi-

\section{REFERÊNCIAS}

1. US Navy. Diving manual. Washington: Navy Department, 1988.

2. Lynch PR. Historical and basic perspectives of scuba diving. Med Sci Sports Exerc 1996;28:570-2.

3. Strozberg MV. Alterações fisiológicas na prática do mergulho. Âmbito Medicina Desportiva 1995;4:5-16.

4. Fox EL, Bowers RW, Foss ML. Bases fisiológicas da educação física e dos desportos. Rio de Janeiro: Guanabara Koogan, 1991.

5. Astrand, Per-Olof, Rodahl K. Tratado de fisiologia do exercício. Rio de Janeiro: Interamericana, 1980.

6. Ministério da Marinha. Manual Didático de Medicina Submarina. Rio de Janeiro: Ciama, 1987.

7. Weineck J. Biologia do esporte. São Paulo: Manole, 1991.

8. Bove AA. Medical aspects of sport diving. Exerc Sport Sci Rev 1996; 28:591-2.

9. Bove AA, Davis J. Diving medicine. Philadelphia: W.B. Saunders, 1990.

10. Flegner AJ, Dias JC. Manual completo de pesquisa e redação. Rio de Janeiro: Esefex, 1995.

11. Araújo CG. Manual de teste de esforço. Rio de Janeiro: Ao livro técnico, 1984 gênio no ar é de aproximadamente $21 \%$ e a pressão parcial é de $0,21 \mathrm{~atm}$. A concentração de oxigênio no ar permanece a mesma durante o mergulho, quando se respira ar atmosférico comprimido, mas a pressão parcial aumenta e reflete a compressão crescente do gás.

O conhecimento sobre as respostas e alterações fisiológicas contribui consideravelmente para a segurança de qualquer modalidade no mergulho. Estas informações são de grande valia para o planejamento detalhado de estratégias voltadas ao estabelecimento de programas de condicionamento e treinamento físico, considerando-se que as demandas físicas no mergulho aumentam em função da pressão hidrostática.

\section{AGRADECIMENTOS}

Agradecemos ao Prof. Dr. Antonio Claudio Lucas da Nóbrega, por sua estimável colaboração na realização deste estudo.

12. Marins JC, Giannichi RS. Avaliação e prescrição de atividade física: guia prático. Rio de Janeiro: Shape, 1996.

13. American College of Sports Medicine. Guidelines for exercise testing and prescription. Philadelphia: Lea \& Febiger, 1991.

14. Hollmann W, Hettinger T. Medicina do esporte. São Paulo: Manole, 1989.

15. West JB. Fisiologia respiratória moderna. São Paulo: Manole, 1996.

16. Terzi RGG. Equilíbrio ácido-básico e transporte de oxigênio. São Paulo: Manole, 1992.

17. Doubt TJ. Cardiovascular and thermal responses to scuba diving. Med Sci Sports Exerc 1996;28:581-6.

18. Kruel LFM, Sampredo RMF. Alterações de freqüência cardíaca no meio aquático - uma revisão. Synopsis. 1996;(7):23-33.

19. Cabric M, Medved R, Denoble P, Zivkovic M, Kovacevic H. Effect of hyperbaric oxygenation on maximal aerobic performance in a normobaric environment. J Sports Med Phys Fitness 1991;31:362-6.

20. Pendergast DR, Tedesco M, Nawrocki DM, Fisher NM. Energetics of underwater swimming with scuba. Med Sci Sports Exerc 1996;28: 573-80. 